\title{
Interhospital Transfer and Receipt of Specialty Procedures
}

\author{
Stephanie K. Mueller, MD, MPH',2*, Jie Zheng ${ }^{3}$, E. John Orav, PhD ${ }^{1,3}$, Jeffrey L. Schnipper, MD, MPH',2
}

'Division of General Internal Medicine, Brigham and Women's Hospital, Boston, Massachusetts; ${ }^{2}$ Harvard Medical School, Boston, Massachusetts; ${ }^{3}$ Harvard T.H. Chan School of Public Health, Boston, Massachusetts.

The practice of transferring patients between acute care hospitals is variable and largely nonstandardized. Although often-cited reasons for transfer include providing patients access to specialty services only available at the receiving institution, little is known about whether and when patients receive such specialty care during the transfer continuum. We performed a retrospective analysis using 2013 100\% Master Beneficiary Summary and Inpatient claims files from Centers for Medicare and Medicaid Services. Beneficiaries were included if they were aged $\geq 65$ years, continuously enrolled in Medicare A and B, with an acute care hospitalization claim, and transferred to another acute care hospital with a primary diagnosis of acute myocardial infarction, gastrointestinal bleed, renal failure, or hip fracture/dislocation. Associated specialty procedure codes (International Classification of Diseases, 9th Revision, Clinical Modification) were identified for each diagnosis. We performed descriptive analyses to compare receipt of specialty procedural services between transferring and receiving hospitals, stratified by diagnosis. Across the 19,613 included beneficiaries, receipt of associated specialty procedures was more common at the receiving than the transferring hospital, with the exception of patients with a diagnosis of gastrointestinal bleed. Depending on primary diagnosis, between $32.4 \%$ and $89.1 \%$ of patients did not receive any associated specialty procedure at the receiving hospital. Our results demonstrate variable receipt of specialty procedural care across the transfer continuum, implying the likelihood of alternate drivers of interhospital transfer other than solely receipt of specialty procedural care. Journal of Hospital Medicine 2018;13:383-387. Published online first November 8, 2017. ( ) 2018 Society of Hospital Medicine

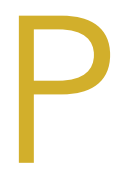

atients who undergo interhospital transfer $(\mathrm{IHT})$ are felt to benefit from receipt of unique specialty care at the receiving hospital. ${ }^{1}$ Although only $1.5 \%$ of all hospitalized Medicare patients undergo hospital transfer, ${ }^{2}$ the frequency of transfer is much greater within certain patient populations, as may be expected with diagnoses requiring specialty care. ${ }^{3,4}$ Existent data demonstrate that $5 \%$ of Medicare patients admitted to the intensive care unit $(\mathrm{ICU})^{5}$ and up to $50 \%$ of patients presenting with acute myocardial infarction (AMI) undergo IHT. ${ }^{6}$

More recent data suggest variability in hospital transfer practices not accounted for by differences in patient or hospital characteristics. ${ }^{2}$ Although disease-specific guidelines for IHT exist for certain diagnoses, ${ }^{3,4}$ the process remains largely nonstandardized for many patients, leading to ambiguity surrounding indications for transfer. Because limited data suggest worse outcomes for transferred versus nontransferred patients, ${ }^{8}$ a better understanding of the specialized care patients

\footnotetext{
*Address for correspondence: Stephanie Mueller, MD, MPH, FHM, Division of General Internal Medicine, Brigham and Women's Hospital, 1620 Tremont Street, Roxbury, MA 02120; Telephone: 617-278-0628; Fax: 617-732-7072; E-mail: smueller1@bwh.harvard.edu
}

Additional Supporting Information may be found in the online version of this article.

Received: May 19, 2017; Revised: July 21, 2017; Accepted: July 25, 2017

๑) 2018 Society of Hospital Medicine DOI 10.12788/jhm.2875 actually receive across the transfer continuum may help to elucidate potential indications for transfer and ultimately help delineate which patients are most (or least) likely to benefit from transfer and why.

In this national study, we examined a select cohort of transferred patients with diagnoses associated with specific specialty procedural services to determine if they received these procedures and where along the transfer continuum they were performed.

\section{METHODS}

We performed a cross-sectional analysis using the Center for Medicare and Medicaid Services 2013 100\% Master Beneficiary Summary and Inpatient claims files. Our study protocol was approved by the Partners Healthcare Human Subjects Review Committee.

Beneficiaries were eligible for inclusion if they were aged $\geq 65$ years, continuously enrolled in Medicare $A$ and $B$, and with an acute care hospitalization claim in 2013, excluding Medicare managed care and end stage renal disease beneficiaries due to incomplete claims data in these groups. We additionally excluded beneficiaries hospitalized at federal or nonacute care hospitals, or critical access hospitals given their mission to stabilize and then transfer patients to referral hospitals. ${ }^{9}$

Transferred patients were defined as beneficiaries with corresponding "transfer in" and "transfer out" claims, or those with either claim and a corresponding date of admission/ discharge from another hospital within 1 day of the claim, as 
we used in our prior research. ${ }^{2}$ Beneficiaries transferred to the same hospital, those with greater than 1 transfer within the same hospitalization, or those cared for at hospitals with "outlier" transfer-in rates equal to $100 \%$ or transfer-out rates greater than $35 \%$ were excluded from analysis given the suggestion of nonstandard claims practices.

We first identified the top 15 primary diagnoses at time of transfer using International Classification of Diseases, Ninth Revision (ICD-9) codes (supplementary Appendix), and then identified those 4 most likely to require specialty procedural services: AMI, gastrointestinal bleed (GI bleed), renal failure, and hip fracture/dislocation. We then chose associated ICD-9 procedure codes for each diagnosis, via expert opinion (authors SM and JS, hospitalist physicians with greater than 20 years of combined clinical experience), erring on overinclusion of procedure codes. We then quantified receipt of associated procedures at transferring and receiving hospitals, stratified by diagnosis.

We further explored the cohort of patients with hip fracture/ dislocation who underwent an associated procedure at the transferring but not receiving hospital, examining the frequency with which these patients had other (nonrelated) procedures at the receiving hospital, and identifying which procedures they received.

\section{RESULTS}

Of the 101,507 patients transferred to another hospital, 19,613 (19.3\%) had a primary diagnosis of AMI, GI bleed, renal failure, or hip fracture/dislocation. Table 1 lists the ICD-9 procedure codes associated with each diagnosis.

Distribution of receipt of specialty procedures at the transferring and receiving hospitals varied by disease (Figure). With the exception of $\mathrm{Gl}$ bleed, patients more often received specialty procedural care at the receiving than the transferring hospital. Depending on primary diagnosis, between $32.4 \%$ and $89.1 \%$ of patients did not receive any associated specialty procedure at the receiving hospital.

Of the 370 (22.1\%) hip fracture/dislocation patients that received a specialty procedure at the transferring but not receiving hospital, 132 (35.7\%) did not receive any procedure at the receiving hospital, whereas the remaining 238 (64.3\%) received an unrelated (not associated with the primary diagnosis) procedure. There was great variety in the types of procedures received, the most common being transfusion of blood products (ICD-9 Clinical Modification 9904).

\section{DISCUSSION}

Among transferred patients with primary diagnoses that have clearly associated specialized procedural services, we found that patients received these procedures at varying frequency and locations across the transfer continuum. Across 4 diagnoses, receipt of associated procedures was more common at the receiving than the transferring hospital, with the exception being patients with GI bleed. We additionally found that many transferred patients did not receive any associated specialty procedure at the receiving hospital. These findings suggest the strong likelihood of more diverse underlying reasons for transfer rather than solely receipt of specialized procedural care.

Despite the frequency with which AMI patients are transferred, ${ }^{6}$ and American Heart Association guidelines directing hospitals to transfer $\mathrm{AMI}$ patients to institutions able to provide necessary invasive treatments, ${ }^{4}$ prior studies suggest these patients inconsistently receive specialty intervention following transfer, including stress testing, cardiac catheterization, or coronary artery bypass graft surgery. ${ }^{10,11}$ Our findings add to these data, demonstrating that only $47.3 \%$ of patients transferred with $\mathrm{AMI}$ received any cardiac-related procedure at the receiving hospital. Additionally, we found that $38.1 \%$ of AMI patients do not receive any specialty procedures at either the transferring or the receiving hospital. Taken together, these data suggest possible discrepancies in the perceived need for these procedures between transferring and receiving hospitals, reasons for transfer related to these conditions that don't involve an associated procedure, or reasons for transfer unrelated to specialty care of the primary diagnosis (such as care of comorbidities, hospital location, prior relationships with that hospital, or desire for a second opinion). Although some of these alternate reasons for transfer likely still benefit the patient, some of these reasons may not justify the increased risks of discontinuity of care created by IHT.

Given limited data looking at IHT practices for patients with other diagnoses, the varying patterns of specialty procedural interventions we observed among transferred patients with $\mathrm{Gl}$ bleed, renal failure, and hip fracture/dislocation are novel contributions to this topic. Notably, we found that among patients transferred with a primary diagnosis of renal failure, the vast majority (84.1\%) did not receive any associated procedure at either the transferring or the receiving hospital. It is possible that although these patients carried the diagnosis of renal failure, their clinical phenotype is more heterogeneous, and they could still be managed conservatively without receipt of invasive procedures such as hemodialysis.

Conversely, patients transferred with primary diagnosis of hip fracture/dislocation were far more likely to receive associated specialty procedural intervention at the receiving hospital, presumably reflective of the evidence demonstrating improved outcomes with early surgical intervention. ${ }^{12}$ However, these data do not explain the reasoning behind the substantial minority of patients who received specialty intervention at the transferring hospital prior to transfer or those that did not receive any specialty intervention at either the transferring or receiving hospital. Our secondary analysis demonstrating great variety in receipt and type of nonassociated procedures provided at the receiving hospital did not help to elucidate potential underlying reasons for transfer.

Notably, among patients transferred with primary diagnosis of GI bleed, receipt of specialty procedures was more common at the transferring (77.7\%) than receiving (63.2\%) hospital, with nearly half (49.3\%) undergoing specialty procedures at both hospitals. It is possible that these findings are reflective of the broad array of specialty procedures examined within this diag- 


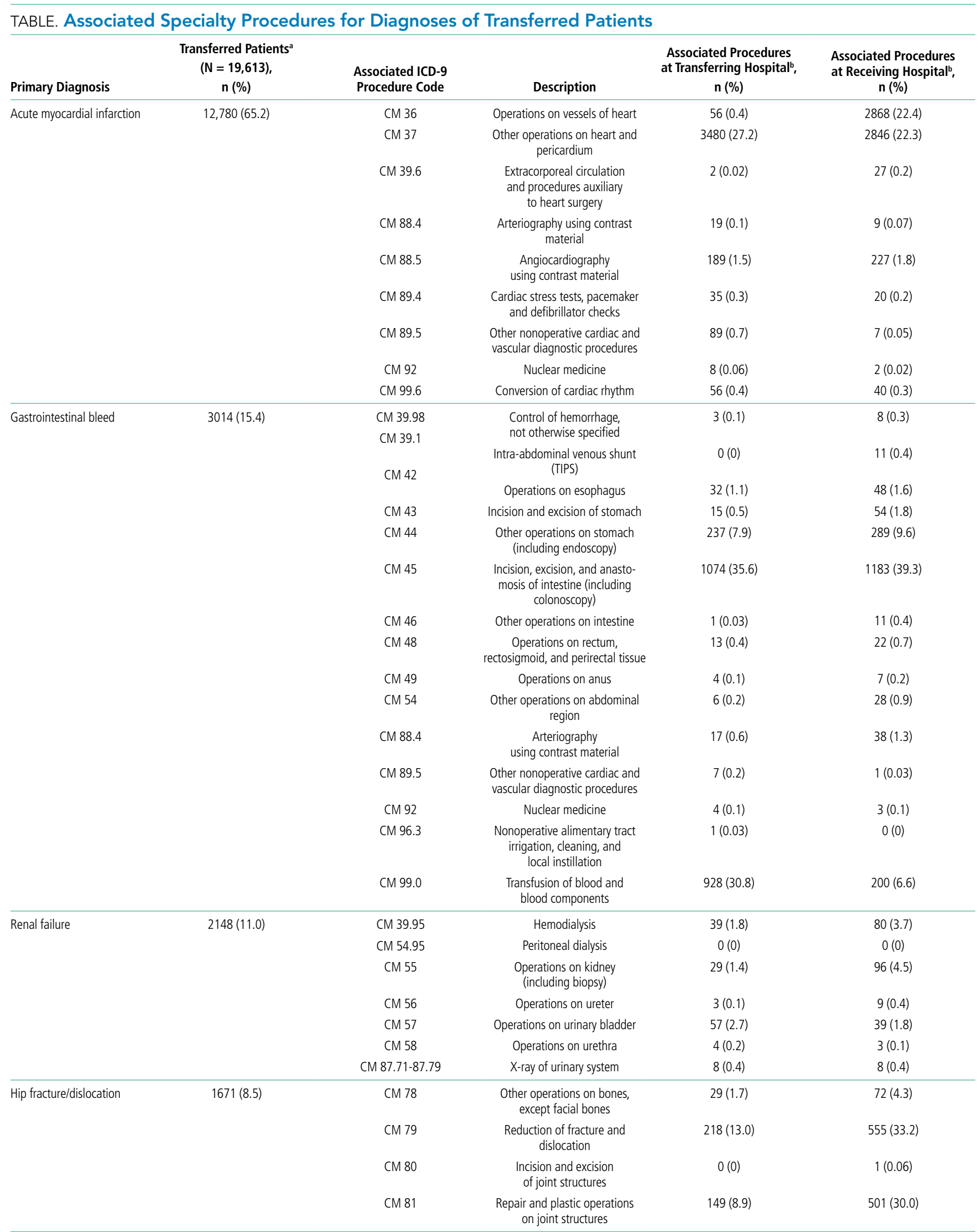

${ }^{a}$ Of the 101,507 transferred patients, 19,613 (19.3\%) had a primary diagnosis of acute myocardial infarction, gastrointestinal bleed, renal failure, or hip fracture/dislocation. Indicates the number of patients receiving each procedure at transferring and receiving hospitals.

NOTE: Abbreviations: CM, Clinical Modification; ICD-9, International Classification of Diseases, Ninth Revision; TIPS, Transjugular Intrahepatic Portosystemic Shunt. 


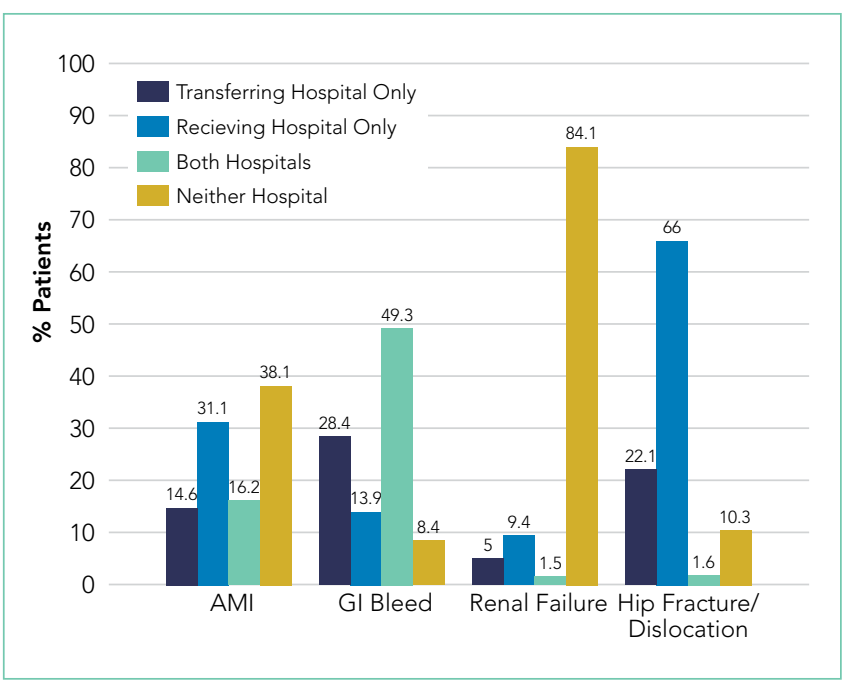

FIG. Frequency of disease-specific procedures at transferring and receiving hospitals.

NOTE: Abbreviations: AMI, acute myocardial infarction; GI, gastrointestinal.

nosis. For example, it is reasonable to consider that a patient may be stabilized with receipt of a blood transfusion at the transferring hospital, then transferred to undergo a diagnostic/therapeutic procedure (ie, endoscopy/colonoscopy) at the receiving hospital, as is suggested by our results.

Our study is subject to several limitations. First, given the criteria we used to define transfer, it is possible that we included nontransferred patients within our transferred cohort if they were discharged from one hospital and admitted to a different hospital within 1 day, although quality assurance analyses we conducted in prior studies on these data support the validity of the criteria used. ${ }^{2}$ Second, we cannot exclude the possibility that patients received nonprocedural specialty care (ie, expert opinion, specialized imaging, medical management, management of secondary diagnoses, etc.) not available at the transferring hospital, although, arguably, in select patients, such input could be obtained without physical transfer of the patient (ie, tele-consult). And even in patients transferred with intent to receive procedural care who did not ultimately receive that care, there is likely an appropriate "nonprocedure" rate, where patients who might benefit from a procedure receive a timely evaluation to reduce the risk of missing the opportunity to receive it. This would be analogous to transferring a patient to an ICU even if they do not end up requiring intubation or pressor therapy. However, given the likelihood of higher risks of IHT compared with intrahospital transfers, one could argue that the threshold of perceived benefit might be different in patients being considered for IHT. Additionally, we limited our analyses to only 4 diagnoses; thus, our findings may not be generalizable to other diagnoses of transferred patients. However, because the diagnoses we examined were ones considered most effectively treated with specialty procedural interventions, it is reasonable to presume that the variability in receipt of specialty procedures observed within these diagnoses is also present, if not greater, across other diagnoses. Third, although we intentionally included a broad array of specialty procedures associated with each diagnosis, it is possible that we overlooked particular specialty interventions. For example, in assuming that patients are most likely to be transferred to receive procedural services associated with their primary diagnosis, we may have missed alternate indications for transfer, including need for procedural care related to secondary or subsequent diagnoses (ie, a patient may have presented with $\mathrm{Gl}$ bleed in the context of profound anemia that requires a bone marrow biopsy for diagnosis, and thus was transferred for the biopsy). Our further examination of unrelated procedures received by hip fracture/dislocation patients at receiving hospitals argues against a select or subset of procedures driving transfers that are not associated with the primary diagnosis but does not fully rule out this possibility (ie, if there are a large variety of secondary diagnoses with distinct associated specialty procedures that are required for each). Lastly, although our examination provides novel information regarding variability in receipt of specialty procedures of transferred patients, we were not able to identify exact reasons for transfer. Instead, our results are hypothesis generating and require further investigation to better understand these reasons.

\section{CONCLUSIONS}

We found that Medicare patients who undergo IHT with primary diagnoses of AMI, Gl bleed, renal failure, and hip fracture/ dislocation receive associated specialty interventions at varying frequency and locations, and many patients do not receive any associated procedures at receiving hospitals. Our findings suggest that specialty procedural care of patients, even those with primary diagnoses that often warrant specialized intervention, may not be the primary driver of IHT as commonly suggested, although underlying reasons for transfer in these and other "nonprocedural" transferred patients remains obscure. Given known ambiguity in the transfer process, ${ }^{7}$ and unclear benefit of $\mathrm{IHT}^{8}$ additional research is required to further identify and evaluate other potential underlying reasons for transfer and to examine these in the context of patient outcomes, in order to understand which patients may or may not benefit from transfer and why.

Disclosure: The authors have nothing to disclose.

\section{References}

1. Iwashyna TJ. The incomplete infrastructure for interhospital patient transfer. Crit Care Med. 2012;40(8):2470-2478.

2. Mueller SK, Zheng J, Orav EJ, Schnipper JL. Rates, Predictors and Variability of Interhospital Transfers: A National Evaluation. J Hosp Med. 2017;12(6):435442.

3. Guidelines for the transfer of critically ill patients. Guidelines Committee of the American College of Critical Care Medicine; Society of Critical Care Medicine and American Association of Critical-Care Nurses Transfer Guidelines Task Force. Crit Care Med. 1993;21(6):931-937.

4. Anderson JL, Adams CD, Antman EM, et al. 2011 ACCF/AHA Focused Update Incorporated Into the ACC/AHA 2007 Guidelines for the Management of Patients With Unstable Angina/Non-ST-Elevation Myocardial Infarction: a report of the American College of Cardiology Foundation/ American Heart Association Task Force on Practice Guidelines. Circulation. 2011;123(18):e426-e579. 
5. Iwashyna TJ, Christie JD, Moody J, Kahn JM, Asch DA. The structure of critical care transfer networks. Med Care. 2009;47(7):787-793.

6. Iwashyna TJ, Kahn JM, Hayward RA, Nallamothu BK. Interhospital transfers among Medicare beneficiaries admitted for acute myocardial infarction at nonrevascularization hospitals. Circ Cardiovasc Qual Outcomes. 2010;3(5):468-475.

7. Bosk EA, Veinot T, Iwashyna TJ. Which patients and where: a qualitative study of patient transfers from community hospitals. Med Care. 2011;49(6):592-598

8. Sokol-Hessner L, White AA, Davis KF, Herzig SJ, Hohmann SF. Interhospita transfer patients discharged by academic hospitalists and general internists: Characteristics and outcomes. J Hosp Med. 2015;11(4):245-250.

9. Department of Health and Human Services, Center for Medicare \& Medicaid
Services: Critical Access Hospitals. https://www.cms.gov/Outreach-and-Education/Medicare-Learning-Network-MLN/MLNProducts/downloads/CritAccessHospfctsht.pdf. Accessed June 29, 2017.

10. Roe MT, Chen AY, Delong ER, et al. Patterns of transfer for patients with nonST-segment elevation acute coronary syndrome from community to tertiary care hospitals. Am Heart J. 2008;156(1):185-192.

11. Barreto-Filho JA, Wang Y, Rathore SS, et al. Transfer rates from nonprocedure hospitals after initial admission and outcomes among elderly patients with acute myocardial infarction. JAMA Intern Med. 2014;174(2):213-222.

12. Doruk H, Mas MR, Yildiz C, Sonmez A, Kyrdemir V. The effect of the timing of hip fracture surgery on the activity of daily living and mortality in elderly. Arch Gerontol Geriatr. 2004;39(2):179-185 